BMJ Open

Diabetes

Research

\& Care

\title{
Decreased risk of colorectal cancer among patients with type 2 diabetes receiving Chinese herbal medicine: a population-based cohort study
}

\author{
Jing-Siang Jhang, ${ }^{1,2}$ Hanoch Livneh, ${ }^{3}$ Shu-Yi Yang, ${ }^{1,2}$ Hui-Ju Huang, ${ }^{4}$ \\ Michael W Y Chan, ${ }^{2,5}$ Ming-Chi Lu, ${ }^{6,7}$ Chia-Chou Yeh, ${ }^{1,8}$ Tzung-Yi Tsai (D) 9,10,11
}

To cite: Jhang J-S, Livneh $\mathrm{H}$, Yang S-Y, et al. Decreased risk of colorectal cancer among patients with type 2 diabetes receiving Chinese herbal medicine: a population-based cohort study. BMJ Open Diab Res Care 2020;8:e000732. doi:10.1136/ bmjdrc-2019-000732

Received 3 July 2019 Revised 11 December 2019 Accepted 4 January 2020
Check for updates

(c) Author(s) (or their employer(s)) 2020. Re-use permitted under CC BY. Published by BMJ.

For numbered affiliations see end of article.

Correspondence to Dr Tzung-Yi Tsai; dm732024@tzuchi.com.tw and Professor Chia-Chou Yeh; yehcc0530@gmail.com

\section{ABSTRACT}

Objectives Patients with type 2 diabetes have a higher risk of colorectal cancer (CRC), but whether Chinese herbal medicines (CHMs) can reduce this risk is unknown. This study investigated the effect that CHMs have on CRC risk in patients with type 2 diabetes.

Research design and methods This cohort study used the Taiwanese National Health Insurance Research Database to identify 54744 patients, newly diagnosed with type 2 diabetes, aged $20-70$ years, who were receiving treatment between 1998 and 2007. From this sample, we randomly selected 14940 CHMs users and 14940 non-CHMs users, using propensity scores matching. All were followed through 2012 to record CRC incidence. Cox proportional hazards regression was used to compute the hazard ratio (HR) of CRC by CHMs use.

Results During follow-up, 235 CHMs users and 375 nonCHMs users developed CRC, incidence rates of $1.73 \%$ and $2.47 \%$ per 1000 person-years, respectively. CHM users had a significantly reduced risk of CRC compared with non-CHM users (adjusted $\mathrm{HR}=0.71 ; 95 \% \mathrm{Cl} 0.60$ to 0.84). The greatest effect was in those receiving $\mathrm{CHMs}$ for more than 1 year. Huang-Qin, Xue-Fu-Zhu-Yu-Tang, ShuJing-Huo-Xue-Tang, Liu-Wei-Di-Huang-Wan, Ji-ShengShen-Qi-Wan, Gan-Lu-Yin, Shao-Yao-Gan-Cao-Tang and Ban-Xia-Xie-Xin-Tang were significantly associated with lower risk of CRC.

Conclusion Integrating CHMs into the clinical management of patients with type 2 diabetes may be beneficial in reducing the risk of CRC.

\section{INTRODUCTION}

Diabetes mellitus has become an increasingly prevalent chronic condition that affects more than 400 million people worldwide. ${ }^{1}$ Over the past 50 years, numerous studies have linked diabetes, in particular type 2 diabetes, to a higher risk of cancer. It is noteworthy that the risk of colorectal cancer (CRC), the third most common cancer around the world, is estimated to be $27 \%$ higher in patients with type 2 diabetes than in non-diabetic control patients. ${ }^{2}$ According to the WHO, approximately 1.8 million patients are newly

\section{Significance of this study}

What is already known about this subject?

- Despite recent improvements in diabetes treatment those with type 2 diabetes are still found to have higher risk of cancer, especially colorectal cancer (CRC).

- Recently, Chinese herbal medicines have become increasingly popular as an adjunctive treatment for patients with chronic diseases; nevertheless, association of Chinese herbal medicines and the risk of CRC among patients with type 2 diabetes is still unknown.

What are the new findings?

- This retrospective 15-year cohort study clarified the therapeutic effect of Chinese herbal medicines on the subsequent risk of CRC among patients with type 2 diabetes.

- The most prominent effect was observed among those receiving Chinese herbal medicines for more than 1 year.

How might these results change the focus of research or clinical practice?

- The reported findings serve an important role for healthcare providers in helping to guide more effective treatment strategies to improve clinical outcomes for patients with type 2 diabetes.

- The corresponding findings could serve as a reference for further pharmacological studies and clinical trials.

diagnosed with CRC, and over 800000 die annually from this cancer. ${ }^{3}$

In Taiwan, CRC is commonly diagnosed and is the third leading cause of cancer-related death. Massive developments in specialized diagnostic and therapeutic methods have improved the survival of patients with type 2 diabetes. Some studies have indicated that the long-term use of insulin or sulfonylureas may predispose patients with type 2 diabetes to develop CRC. ${ }^{45}$ In view of this concern, it is 
of therapeutic interest to explore alternative treatments that may lessen this risk.

With few side effects, Chinese herbal medicines (CHMs) had been used for patients with chronic illnesses such as dementia, ${ }^{6}$ hepatocellular carcinoma ${ }^{7}$ and vertigo. ${ }^{8}$ Several randomized clinical trials have further suggested that CHMs could delay the progression of distal symmetric polyneuropathy ${ }^{9-11}$ and nephropathy. ${ }^{12}$ More specifically, research findings have suggested that CHMs protect against CRC risk by improving the antitumor activity of the chemotherapy drug 5-fluorouracil against the HT-29 colon cancer cell, ${ }^{13}$ implying that CHMs should not be neglected in the treatment of patients with type 2 diabetes.

Given the possible beneficial effect of CHMs on subsequent CRC risk, and the limited information on whether CHMs could modify the relationship between type 2 diabetes and CRC risk, findings from a long-term population-based cohort study might be useful in allocating medical resources and in instituting fact-based policymaking for the treatment of CRC among patients with type 2 diabetes. Nevertheless, to date, no clinical observations or empirical data have documented this potential benefit for patients with type 2 diabetes. Therefore, in this study, we analyzed a nationwide populationbased database to compare the risk of CRC among patients with type 2 diabetes who either did or did not receive CHMs.

\section{RESEARCH DESIGN AND METHODS}

\section{Data source}

For this study, we used a publicly released cohort dataset, the Longitudinal Health Insurance Database (LHID), comprised of approximately 1000000 randomly sampled people and obtained all records from 1996 to 2012. The database has been confirmed by the National Health Research Institute to be representative of the Taiwanese population and its data have been used in many previously published scientific papers. ${ }^{14}$ The encrypted information protects patient privacy and allows linkage of all claims for the same patient within the database. This database contains all National Health Insurance (NHI) enrolment files, claims data and the registry for prescription drugs and provides comprehensive information on all individuals covered by the insurance program. This study was conducted in accordance with the Helsinki Declaration and was evaluated and approved by the local Institutional Review Board and ethics committee of Buddhist Dalin Tzu Chi Hospital, Taiwan (No. B10004021-2).

\section{Study population}

Patients, 20-70 years of age, newly diagnosed with type 2 diabetes between 1998 and 2007 were identified (figure 1). To be included, patients with type 2 diabetes had to have at least three ambulatory or inpatient claims with the International Classification of Disease, 9th edition, Clinical Modification (ICD-9-CM) diagnosis

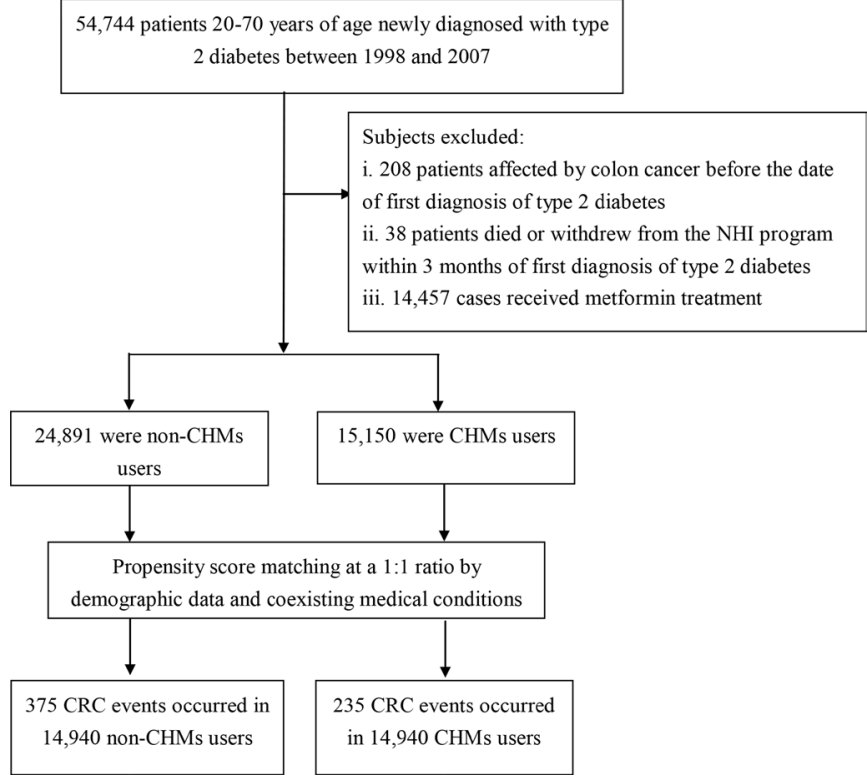

Figure 1 Flowchart of study and follow-up of study participants. CHMs, Chinese herbal medicines; NHI, National Health Insurance.

code 250 and excluding type 1 diabetes (ICD-9-CM code 2501). A total of 208 cases with type 2 diabetes were then excluded because of prior diagnosis of CRC (ICD-9-CM codes colon cancer: 153x, rectal cancer: $154 \mathrm{x})$. This exclusion was indicated by linking type 2 diabetes subjects to the catastrophic illness registry. In Taiwan, insured residents with major diseases (eg, cancer, autoimmune diseases, chronic renal failure) can apply for a catastrophic illness certificate that grants exemption from copayment. Those with a follow-up period $<3$ months were also excluded $(n=38)$. Additionally, given the protective effect of metformin in decreasing the onset of cancer, ${ }^{15}$ those patients who received in the past metformin with an average dose of more than $250 \mathrm{mg}$ per day were also excluded $(\mathrm{n}=14457)$. Overall, we identified 40041 subjects with new-onset type 2 diabetes.

In Taiwan, only certified Chinese medicine physicians are entitled to prescribe CHMs. We used the frequency of visits to a Chinese medicine physician to verify the CHMs exposure of each subject with type 2 diabetes. Those receiving CHMs after their initial diagnosis of type 2 diabetes for more than 30 days were identified as CHMs users, whereas those treated for 30 days or less were considered non-CHMs users. ${ }^{8}$ Based on this rule, 15150 cases were designated as CHMs users. A comparison cohort was randomly selected from the remaining insured type 2 diabetes cases without CHMs use. For each type 2 diabetes case who received CHMs treatment, one control case without CHMs treatment was selected, using a 1:1 match based on a propensity score. Propensity scores representing the likelihood of receiving CHMs were calculated using logistic regression analysis, conditional on the baseline covariates listed in table 1. Accordingly, an equal number of patients in the CHMs and non-CHMs cohorts were analyzed in this study. The index date for 
Table 1 Demographic data and comorbidity comparison of the study subjects

\begin{tabular}{|c|c|c|c|}
\hline & $\begin{array}{l}\text { CHMs non- } \\
\text { users }\end{array}$ & CHMs users & \\
\hline Variables & $n=14940(\%)$ & $n=14940(\%)$ & value \\
\hline Age, (years) & & & 0.75 \\
\hline$\leq 50$ & 5259 (35.2) & $5274(35.3)$ & \\
\hline$>50$ & 9681 (64.8) & 9666 (64.7) & \\
\hline Mean (SD) & $53.93 \pm 10.32$ & $53.86 \pm 10.42$ & 0.74 \\
\hline Gender & & & 0.50 \\
\hline Female & 8396 (56.2) & $8337(55.8)$ & \\
\hline Male & $6544(43.8)$ & 6603 (44.2) & \\
\hline Monthly income & & & 0.51 \\
\hline Low & 5692 (38.1) & 5677 (38.0) & \\
\hline Median & $8411(56.3)$ & $8381(56.1)$ & \\
\hline High & 837 (5.6) & $882(5.8)$ & \\
\hline Residential area & & & 0.28 \\
\hline Urban & $8725(58.4)$ & 8650 (57.9) & \\
\hline Suburban & $2256(15.1)$ & $2346(15.7)$ & \\
\hline Rural & 3959 (26.5) & 3944 (26.5) & \\
\hline \multicolumn{4}{|l|}{ Comorbidity } \\
\hline Hypertension & 6917 (46.3) & $6872(46.0)$ & 0.49 \\
\hline Stroke & $1121(7.5)$ & $1150(7.7)$ & 0.53 \\
\hline Heart disease & 2928 (19.6) & 2928 (19.6) & 0.99 \\
\hline $\begin{array}{l}\text { Chronic kidney } \\
\text { disease }\end{array}$ & $194(1.3)$ & 194 (1.3) & 0.67 \\
\hline Depression & $493(3.3)$ & $538(3.6)$ & 0.16 \\
\hline $\begin{array}{l}\text { Rheumatologic } \\
\text { disorders }\end{array}$ & $105(0.7)$ & $105(0.7)$ & 0.77 \\
\hline
\end{tabular}

CHMs, Chinese herbal medicines.

the follow-up period for non-CHMs users was the date of the type 2 diabetes diagnosis; the index date for the follow-up period for CHMs users was the first date of the initiation of CHMs service. The end date of the follow-up period for both groups was the earliest of the following: (1) having a diagnosis of CRC; (2) withdrawing from the insurance program or (3) the date of December 31, 2012.

\section{Covariate assessment}

Covariates comprised the baseline sociodemographic characteristics and comorbidities. Sociodemographic data included patient's age, sex, income (for estimating insurance payments) and urbanization level of the residential area. Monthly income was stratified into New Taiwan Dollar (NTD) $\leq 17880$; NTD 17 881-NTD 43900 and $\geq$ NTD 43 901. Urbanization levels were divided into urban (levels 1-2), suburban (levels 3-4) and rural (levels $5-7)$. Level 1 refers to the "most urbanized" communities and level 7 refers to the "least urbanized". ${ }^{16}$ Baseline comorbidities included hypertension (ICD-9-CM 401-405), stroke (ICD-9-CM 430-438), heart disease (ICD-9-CM 410-429), chronic kidney disease (ICD-9-CM
585), depression (ICD-9-CM 296.2, 296.3, 300.4 and 311) and rheumatologic disorders (ICD-9-CM 725-729); all were based on each individual's medical records for the 1 year prior to initial cohort entry.

\section{Statistical analysis}

We conducted $\chi^{2}$ test and $t$-test analyses to examine the differences in demographic characteristics and comorbidities between patients with type 2 diabetes with and without CHMs treatment. The incidence rate of CRC was calculated as the number of cases per 1000 person-years (PYs). Multivariate Cox proportional hazards regression analysis was then applied to compute the HR with $95 \%$ CI of CRC risk in association with CHMs use. To test the robustness of the relationship between CHMs use and subsequent CRC risk, we divided the CHMs users into two subgroups: those who used CHMs for 30-365 days and those who used CHMs for more than 365 days. Analysis stratified by age and sex using Cox proportional hazards regression was also conducted to assess the HR of CRC among those who did and did not receive CHMs. Assumptions of the proportional hazards model were verified by using plots of $\log$ (-log (survival)) function) versus $\log$ (time) and Schoenfeld residuals versus time. All analyses were conducted using SAS V.9.3 software (SAS Institute, Cary, North Carolina, USA), at a $\mathrm{p}<0.05$ statistically significant level.

\section{RESULTS}

The CHMs user and non-CHMs user cohorts provided, each, data for 14940 subjects. After the matching procedure with propensity score, no significant differences were observed between the two groups on age, sex, monthly income, residential area and comorbidities, indicating that the two groups were comparable with regard to these variables (table 1 ).

Among all eligible subjects, 610 first episodes of CRC occurred, 375 in non-CHMs users and 235 in CHMs users, during follow-up periods of 151745.04 and 135,251.86 PYs, respectively. The incidence rate of CRC was significantly lower in CHMs users than in non-CHMs users (1.73 vs 2.47 , respectively, per $1000 \mathrm{PYs}$ ), with an adjusted HR of 0.71 (95\% CI 0.60 to 0.84 ) (table 2). Of note, those who used CHMs for more than 365 days had a lower risk of CRC ( $95 \%$ CI 0.28 to 0.61 ) vs non-users by $59 \%$.

Table 3 presents the results from this analysis stratified by age and gender. Collectively, a more significant beneficial effect of CHMs was observed among younger subjects, irrespective of sex. Furthermore, the multivariable stratified analysis verified that the benefit of CHMs therapy for treating CRC was more predominant in males than in females, with an adjusted HR of 0.66 and 0.76 , respectively (table 3 ). The 10 most commonly prescribed herbal formulae for those with type 2 diabetes are summarized in table 4. Among them, one was a single-herb product while the remaining products were multiple-herb products. Cox proportional hazards regression analysis 
Table 2 Crude and adjusted HR of colorectal cancer for patients with type 2 diabetes with and without CHMs treatment

\begin{tabular}{llllll}
\hline Patient group & Event & PYs & Incidence & $\begin{array}{l}\text { Crude HR } \\
\text { (95\% Cl) }\end{array}$ & $\begin{array}{l}\text { Adjusted HR } \\
\text { (95\% Cl) }\end{array}$ \\
\hline CHMs non-users & 375 & 151745.04 & 2.47 & 1.00 & 1 \\
CHMs users & 235 & 135251.86 & 1.73 & $0.70(0.60$ to 0.83$)$ & $0.71(0.60$ to 0.84$)$ \\
$\quad$ CHMs use for 31-365 days & 208 & 108166.55 & 1.92 & $0.78(0.66$ to 0.93$)$ & $0.79(0.67$ to 0.94$)$ \\
CHMs use >1 year & 27 & 27085.31 & 1.00 & $0.40(0.27$ to 0.60$)$ & $0.41(0.28$ to 0.61$)$ \\
\hline
\end{tabular}

Incidence rate is per 1000 PYs.

*Model adjusted for age, gender, urbanization level, monthly income and comorbidity.

CHMs, Chinese herbal medicines; PYs, patient-years.

showed that the use of Huang-Qin, Shu-Jing-Huo-XueTang, Xue-Fu-Zhu-Yu-Tang, Ji-Sheng-Shen-Qi-Wan, LiuWei-Di-Huang-Wan, Gan-Lu-Yin, Shao-Yao-Gan-Cao-Tang and Ban-Xia-Xie-Xin-Tang was significantly associated with lower risk of CRC (table 4).

\section{DISCUSSION}

This is the first evidence-based cohort study addressing the association between CHMs use and CRC risk in patients with type 2 diabetes through a large nationwide claims-based data source. In this follow-up study of 15 years (1998-2012), we found that patients with type 2 diabetes who were receiving CHMs had a nearly $30 \%$ lower chance of CRC than those not using CHMs. Furthermore, those receiving CHMs for more than 365 days were found to have a lower risk of CRC by nearly $60 \%$. The dose-response relationship may elucidate the causality between CHMs use and the decrease in the predisposition for developing CRC. No previous studies have been conducted to determine the longtern impact of CHMs on CRC risk among patients with type 2 diabetes, thus rendering a comparison of results impossible. However, the findings obtained herein are consistent with earlier research findings and add to the growing body of knowledge on the beneficial effects of CHMs for the patients with chronic diseases. ${ }^{6-8}$

The effect of CHMs was further analyzed with stratification by age and sex. Taken together, the use of CHMs was found to have a greater effect on CRC risk in younger patients irrespective of their sex, and th finding echod these of previous studies. ${ }^{78}$ We speculated that younger patients may have fewer coexisting medical conditions or possess better medical knowledge and coping resources, in addition to demonstrating more positive attitudes toward medical impairments, ${ }^{17}$ thus enhancing the preventive impact of CHMs on CRC risk.

An additional contribution of the present study is the list of Chinese herbal products that were found to be beneficial in reducing CRC risk. For example, Huang-Qin is one of the most common CHMs used to treat type 2 diabetes and it may help in lessening the subsequent risk for CRC. Wogonin, the main ingredient in Huang-Qin, was found to have antineoplastic and antiinflammatory effects in both in vitro and in vivo studies. One study found that wogonin can induce activation of AMP-activated protein kinase (AMPK), to inhibit the proliferation and induce apoptosis in cancer cells. ${ }^{18}$ Additionally, other researchers have proposed that wogonin

Table 3 Age-specific and sex-specific incidence and adjusted HR of colorectal cancer in relation to CHMs use in patients with type 2 diabetes

\begin{tabular}{|c|c|c|c|c|c|c|c|c|}
\hline \multirow[b]{2}{*}{ Variables } & \multicolumn{3}{|c|}{ CHMs non-users } & \multicolumn{3}{|c|}{ CHMs users } & \multirow[b]{2}{*}{ Crude HR $(95 \%$ Cl) } & \multirow{2}{*}{$\begin{array}{l}\text { Adjusted HR } \\
(95 \% \mathrm{Cl})\end{array}$} \\
\hline & Case & PYs & Incidence & Case & PYs & Incidence & & \\
\hline \multicolumn{9}{|l|}{ Female } \\
\hline$>50$ years & 154 & 58674.38 & 2.62 & 107 & 48944.48 & 2.19 & 0.84 (0.66 to 1.07$)$ & $0.84(0.65 \text { to } 1.07)^{*}$ \\
\hline All & 187 & 84775.18 & 2.21 & 124 & 75221.5 & 1.65 & 0.76 (0.59 to 0.94$)$ & $0.76(0.61$ to 0.96$) \dagger$ \\
\hline$\leq 50$ years & 39 & 25323.32 & 1.54 & 20 & 22241.56 & 0.90 & 0.60 (0.35 to 0.98$)$ & $0.59(0.34 \text { to } 0.97)^{\star}$ \\
\hline$>50$ years & 149 & 41646.54 & 3.58 & 91 & 37788.8 & 2.41 & 0.67 (0.52 to 0.87$)$ & $0.68(0.52 \text { to } 0.88)^{*}$ \\
\hline All & 188 & 66969.86 & 2.81 & 111 & 60030.36 & 1.85 & 0.65 (0.52 to 0.83$)$ & $0.66(0.53$ to 0.84$) \dagger$ \\
\hline
\end{tabular}

Incidence rate is per 1000 PYs.

*Model adjusted for urbanization level, monthly income and comorbidity.

†Model adjusted for age, urbanization level, monthly income and comorbidity.

CHMs, Chinese herbal medicines; PYs, person-years. 
Table 4 Risk of colorectal cancer in relation to the 10 most commonly used CHMs for patients with type 2 diabetes

\begin{tabular}{|c|c|c|c|c|}
\hline CHMs name & Ingredients or generic name & $\begin{array}{l}\text { Frequency of } \\
\text { prescriptions }\end{array}$ & $\begin{array}{l}\text { Crude HR } \\
(95 \% \mathrm{Cl})\end{array}$ & $\begin{array}{l}\text { Adjusted HR * } \\
(95 \% \mathrm{Cl})\end{array}$ \\
\hline \multicolumn{5}{|l|}{ Single-herb products } \\
\hline Huang-Qin & Scutellariae radix & 15796 & $\begin{array}{l}0.67 \\
(0.52 \text { to } 0.84)\end{array}$ & $\begin{array}{l}0.68 \\
(0.56 \text { to } 0.90)\end{array}$ \\
\hline \multicolumn{5}{|l|}{ Multiherb products } \\
\hline $\begin{array}{l}\text { Shu-Jing-Huo- } \\
\text { Xue-Tang }\end{array}$ & $\begin{array}{l}\text { Paeoniae radix, Angelicae radix, Atractylodis lanceae rhizome, Cnidii } \\
\text { rhizome, Persicae semen, Poria, Rehmanniae radix, Achyranthis } \\
\text { radix, Clematis radix, Gentianae scabrae radix, Notoptergii rhizome, } \\
\text { Saposhnikoviae radix, Sinomeni caulis et rhizome, Angelicae dahuricae radix, } \\
\text { Aurantii nobillis pericarpium, Glycyrrhizae radix, Zingiberis rhizoma }\end{array}$ & 18503 & $\begin{array}{l}0.65 \\
(0.54 \text { to } 0.78)\end{array}$ & $\begin{array}{l}0.69 \\
(0.56 \text { to } 0.81)\end{array}$ \\
\hline $\begin{array}{l}\text { Xue-Fu-Zhu-Yu- } \\
\text { Tang }\end{array}$ & $\begin{array}{l}\text { Glycyrrhizae radix, Angelicae sinensis radix, Rehmanniae radix, Achyranthis } \\
\text { bidentatae radix, Persicae semen, Chuanxiong rhizoma, Platycodonis radix, } \\
\text { Carthami flos, Paeoniae radix rubra, Bupleuri radix, Aurantii fructus }\end{array}$ & 11664 & $\begin{array}{l}0.56 \\
(0.44 \text { to } 0.72)\end{array}$ & $\begin{array}{l}0.60 \\
(0.46 \text { to } 0.76)\end{array}$ \\
\hline $\begin{array}{l}\text { Ji-Sheng-Shen- } \\
\text { Qi-Wan }\end{array}$ & $\begin{array}{l}\text { Rehmanniae radix preparata, Fructus corni, Cortex moutan, Rhizoma } \\
\text { dioscoreae, Poria and Rhizoma alismatis, Achyranthis bidentatae radix, } \\
\text { Aconiti lateralis praeparata radix, Cinnamomi cortex, Plantaginis semen }\end{array}$ & 12498 & $\begin{array}{l}0.55 \\
(0.42 \text { to } 0.72)\end{array}$ & $\begin{array}{l}0.53 \\
(0.40 \text { to } 0.69)\end{array}$ \\
\hline $\begin{array}{l}\text { Liu-Wei-Di-Huang- } \\
\text { Wan }\end{array}$ & $\begin{array}{l}\text { Rehmanniae radix preparata, Fructus corni, Cortex moutan, Rhizoma } \\
\text { dioscoreae, Poria, Rhizoma alismatis }\end{array}$ & 11031 & $\begin{array}{l}0.58 \\
(0.49 \text { to } 0.67)\end{array}$ & $\begin{array}{l}0.62 \\
(0.53 \text { to } 0.72)\end{array}$ \\
\hline Gan-Lu-Yin & $\begin{array}{l}\text { Glycyrrhilza uralensis, Liriope spicata, Citrus sinensis, Rehmannia glutinose, } \\
\text { Artemisia capillaris, Eriobotrya japonica, Dendrobium nobile, Scutellaria } \\
\text { baicalensis and Asparagus cochinchinensis }\end{array}$ & 9753 & $\begin{array}{l}0.62 \\
(0.47 \text { to } 0.81)\end{array}$ & $\begin{array}{l}0.64 \\
(0.49 \text { to } 0.84)\end{array}$ \\
\hline $\begin{array}{l}\text { Du-Huo-Ji-Sheng } \\
\text { Tang }\end{array}$ & $\begin{array}{l}\text { Angelicae pubescentis radix, Taxilli herba, Eucommiae cortex, Cyathulae } \\
\text { radix, Asari radix, Gentianae macrophyllae radix, Poria, Cinnamomi cortex, } \\
\text { Saposhnikoviae radix, Chuanxiong rhizoma, Ginseng radix, Glycyrrhizae } \\
\text { radix, Angelicae sinensis radix, Paeoniae radix alba, Rehmanniae radix }\end{array}$ & 9063 & $\begin{array}{l}0.79 \\
(0.64 \text { to } 0.97)\end{array}$ & $\begin{array}{l}0.76 \\
(0.61 \text { to } 1.01)\end{array}$ \\
\hline $\begin{array}{l}\text { Shao-Yao-Gan- } \\
\text { Cao-Tang }\end{array}$ & Paeoniae radix, Glycyrrhizae radix & 8003 & $\begin{array}{l}0.74 \\
(0.60 \text { to } 0.91)\end{array}$ & $\begin{array}{l}0.75 \\
(0.57 \text { to } 0.89)\end{array}$ \\
\hline Yu-Quan-Wan & $\begin{array}{l}\text { Ophiopogonis radix, Ginseng radix, Poria, Astragali radix, Mume fructus, } \\
\text { Trichosanthis fructus, Glycyrrhizae radix, Puerariae lobatae radix }\end{array}$ & 4932 & $\begin{array}{l}0.88 \\
(0.56 \text { to } 1.13)\end{array}$ & $\begin{array}{l}0.92 \\
(0.60 \text { to } 1.23)\end{array}$ \\
\hline $\begin{array}{l}\text { Ban-Xia-Xie-Xin- } \\
\text { Tang }\end{array}$ & $\begin{array}{l}\text { Pinelliae rhizoma, Scutellariae radix, Zingiberis rhizoma, Ginseng radix, } \\
\text { Glycyrrhizae radix, Coptidis rhizoma, Jujubae fructus }\end{array}$ & 4529 & $\begin{array}{l}0.69 \\
(0.52 \text { to } 0.91)\end{array}$ & $\begin{array}{l}0.70 \\
(0.54 \text { to } 0.97)\end{array}$ \\
\hline
\end{tabular}

*Model adjusted for age, gender, urbanization level, monthly income and comorbidities.

$\mathrm{CHMs}$, Chinese herbal medicines.

may suppress the production of interleukin (IL)-6 and IL-1b by modulating the nuclear factor (NF)-kappa beta and NF-E2-related factor 2 signaling pathways. ${ }^{19}$ IL- 6 and IL-1 $\beta$ are well known pleiotropic proinflammatory cytokines with profound effects on several diseases, especially CRC onset. ${ }^{2021}$

Findings of the present study revealed that the other commonly-prescribed formulas targeting type 2 diabetes, such as Xue-Fu-Zhu-Yu-Tang and Shu-Jing-Huo-XueTang, also significantly lowered CRC risk. Recently, both animal experiments and human studies showed that Xue-Fu-Zhu-Yu-Tang has anti-inflammatory and antitumor effects, primarily via inhibiting the activation of such intracellular signaling pathways as phosphoinositide 3-kinase-protein kinase B-mammalian target of rapamycin (PI3K-AKT-mTOR). ${ }^{22} 23$ Blocking PI3K-AKT-mTOR signaling may impact the expression levels of elements in the tumor microenvironment, affecting cancer stem cell expression and survival. ${ }^{24}$ In addition, the positive therapeutic effect of Shu-Jing-Huo-Xue-Tang on subsequent risk of CRC may be inferred from its reported effect of increasing blood circulation and enhancing antioxidant enzymatic activity, ${ }^{25}$ in addition to its anti-inflammatory activity. ${ }^{26}$ All of these processes have been implicated in the development of colitis-associated neoplasia. ${ }^{27}$

Similar to a previous report, ${ }^{28}$ the current study showed a preventive effect of Liu-Wei-Di-Huang-Wan on the risk of CRC. This herbal product may induce the inactivation of the IGF pathway. IGF is well known to promote malignant transformation, promoting cell proliferation and dedifferentiation and inhibiting apoptosis, which in turn predisposes the individual to developing cancer. ${ }^{29}$ Ji-Sheng-Shen-Qi-Wan, also known as Gosha-jinki-gan, is widely used to treat patients with diabetic neuropathy. This medication enhances nitric oxide $(\mathrm{NO})$ production and thereby increases blood circulation while inhibiting blood coagulation. ${ }^{30}$ Recently, NO has been suggested to modulate different cancer-related events, including angiogenesis, apoptosis and metastasis, ${ }^{31}$ all possible mechanisms for the positive effect observed here.

Gan-Lu-Yin was also found to decrease the risk of CRC. In one study, relative to untreated controls, Gan-LuYin-fed rats had markedly reduced cell proliferation and migration, through the induced differentiation of WEHI-3 cells. ${ }^{32}$ This inhibition of angiogenesis may prevent subsequent tumor growth. Use of another common CHMs, 
Shao-Yao-Gan-Cao-Tang, was also related to a lower risk of CRC. This compound may rescue the decreased phosphorylation of glycogen synthase kinase 3 (GSK-3) in patients with type 2 diabetes. ${ }^{33}$ Activation of GSK-3 would then downregulate the PI3K-AKT-mTOR signaling pathway to inhibit colon cancer cell proliferation. ${ }^{34}$

Use of Ban-Xia-Xie-Xin-Tang was found to lessen the risk of developing CRC in patients with type 2 diabetes. One possible mechanism may involve the anti-inflammatory properties of Ban-Xia-Xie-Xin-Tang, ${ }^{35} 36$ which is often used to treat various digestive inflammations, like colitis, esophagitis and gastritis. ${ }^{37}$ Chronic inflammation of the bowel has been hypothesized as the most important mechanism driving CRC onset. ${ }^{38}$

While our study is the first to investigate CHMs effect on reducing CRC risk among patients with type 2 diabetes, there are important limitations to consider. First, a coding error may occur in the retrospective study design due to factors related to the availability and accuracy of the medical record. Therefore, we enrolled patients with type 2 diabetes and CRC only after these patients had at least three outpatient visits reporting consistent diagnoses, or after the patients had at least one inpatient admission. The CRC cases were further verified using the catastrophic illness registry. It should also be noted that the Taiwan NHI randomly audits hospital claims, interviews patients and reviews medical charts to verify the accuracy of medical records. Second, the LHID does not include detailed information on the risk factors associated with CRC, such as smoking, alcohol consumption, level of obesity or dietary habits. Future research, considering these untested variables, is needed to better assess whether the present findings are replicable across diverse groups of individuals. Third, prescriptions for medications issued before 1996 were not reflected in our data analysis. This omission may have resulted in underestimation of the cumulative frequency of prescriptions and therefore may have weakened the apparent effects of the specified herbal products. Although several commonly-prescribed CHMs for patients with type 2 diabetes were identified as influential in decreasing the risk of CRC, their therapeutic effect and safety concerns remain to be elucidated in future pharmacological investigations. Fourth, we conducted two sensitivity analysis to confirm the relationships of interest. First, we did not exclude those receiving metformin with an average dose of more than $250 \mathrm{mg}$ per day $(\mathrm{n}=14457)$. The reanalysis showed that the protective effect of CHMs was somewhat attenuated, but was still statistically significant (adjusted $\mathrm{HR}=0.76 ; 95 \%$ CI 0.64 to $0.89)$. Second, all CRC cases were divided into two groups based on the location of CRC, namely, the occurrence of malignant neoplasm of colon and rectal cancer. Of the 610 CRC cases, 395 were of colon cancer and 215 cases were attributed to rectal cancer. After adjusting for the confounders, the reanalysis indicated that integrating CHMs into the conventional therapy decreased the risk of colon cancer and rectal cancer, with an adjusted HR of 0.77 (95\% CI 0.64 to 0.95 ) and 0.60 (95\% CI 0.45 to
0.79), respectively. Taken together, the results from the reanalysis of the two sensitivity analyses lent support to the earlier findings of this study. Fifth, although our study revealed a substantial benefit resulting from the use of CHMs among patients with type 2 diabetes, it must be recognized that in our study patients were not randomly categorized into users and nonusers of CHMs. Therefore, potential biases may still remain, and in particular when they stem from unmeasured or unknown confounders. Caution, therefore, must be exercised when interpreting these findings.

These limitations notwithstanding, this study also offer several strengths. These include the immediate availability of data, the comprehensiveness of the database, and the statistical power derived from the large sample. In addition, a long observational period offers the opportunity to determine in detail the association between CHMs usage and CRC risk among patients with type 2 diabetes, and the available findings may serve as a useful reference for future studies focusing on additional clinical outcomes, such as colorectal neoplasm.

\section{CONCLUSION}

In summary, this large-scale nationwide cohort study demonstrates that the integration of CHMs, during treatment for type 2 diabetes, appears to lower the subsequent risk of CRC by nearly $30 \%$. We believe that these findings could serve as a reference for healthcare providers in helping to establish more effective therapeutic interventions to improve the prognosis of patients with type 2 diabetes.

\section{Author affiliations}

${ }^{1}$ Department of Chinese Medicine, Dalin Tzuchi Hospital, The Buddhist Tzuchi Medical Foundation, Chiayi, Taiwan

${ }^{2}$ Department of Biomedical Sciences, National Chung Cheng University, Chiayi, Taiwan

${ }^{3}$ Rehabilitation Counseling Program, Portland State University, Portland, Oregon, USA

${ }^{4}$ Department of Nursing, Dalin Tzuchi Hospital, The Buddhist Tzuchi Medical Foundation, Chiayi, Taiwan

${ }^{5}$ Epigenomics and Human Diseases Research Center, National Chung Cheng University, Chiayi, Taiwan

${ }^{6}$ Division of Allergy, Immunology and Rheumatology, Dalin Tzuchi Hospital, The Buddhist Tzuchi Medical Foundation, Chiayi, Taiwan

${ }^{7}$ School of Medicine, Tzu Chi University, Hualien, Taiwan

${ }^{8}$ School of Post-Baccalaureate Chinese Medicine, Tzu Chi University, Hualien, Taiwan

${ }^{9}$ Department of Environmental and Occupational Health, College of Medicine, National Cheng Kung University, Tainan, Taiwan

${ }^{10}$ Department of Nursing, Tzu Chi University of Science and Technology, Hualien, Taiwan

${ }^{11}$ Department of Medical Research, Dalin Tzuchi Hospital, The Buddhist Tzuchi Medical Foundation, Chiayi, Taiwan

Acknowledgements The study is based in part on data from the National Health Insurance Research Database provided by the Bureau of National Health Insurance, Department of Health and managed by the National Health Research Institutes, in Taiwan. The interpretation and conclusions contained herein do not represent those of the Bureau of National Health Insurance, Department of Health or National Health Research Institutes. JSJ, HL, SYY, HJH and MWYC contributed equally to this work. 
Contributors JSJ, SYY and TYT were involved in the study design and drafted the manuscript. HL and TYT contributed to data analysis and revised the manuscript. MCL, HJH, MWYC and CCY contributed to the interpretation of data and provided comments on the final draft of the manuscript. MCL and CCY provided administrative support and comments on the manuscript drafts. TYT, HL and JSJ were responsible for the study conception, design, data analysis and drafting. All authors read and approved the final manuscript.

Funding The authors have not declared a specific grant for this research from any funding agency in the public, commercial or not-for-profit sectors.

Competing interests None declared.

Patient consent for publication Not required.

Ethics approval This study was approved by the Research of Ethics Committee of Dalin Tzuchi Hospital (No. B10004021-2).

Provenance and peer review Not commissioned; externally peer reviewed.

Data availability statement № data are available.

Open access This is an open access article distributed in accordance with the Creative Commons Attribution 4.0 Unported (CC BY 4.0) license, which permits others to copy, redistribute, remix, transform and build upon this work for any purpose, provided the original work is properly cited, a link to the licence is given, and indication of whether changes were made. See: https://creativecommons.org/ licenses/by/4.0/.

ORCID iD

Tzung-Yi Tsai http://orcid.org/0000-0002-9865-7101

\section{REFERENCES}

1 Ogurtsova K, da Rocha Fernandes JD, Huang Y, et al. IDF diabetes atlas: global estimates for the prevalence of diabetes for 2015 and 2040. Diabetes Res Clin Pract 2017;128:40-50.

2 González N, Prieto I, Del Puerto-Nevado L, et al. 2017 update on the relationship between diabetes and colorectal cancer: epidemiology, potential molecular mechanisms and therapeutic implications. Oncotarget 2017;8:18456-85.

3 McGuire S. World cancer report 2014. Geneva, Switzerland: World Health organization, International agency for research on cancer, who press, 2015. Adv Nutr 2016;7:418-9.

4 Chang C-H, Lin J-W, Wu L-C, et al. Oral insulin secretagogues, insulin, and cancer risk in type 2 diabetes mellitus. J Clin Endocrinol Metab 2012;97:E1170-5.

5 Yang Y-X, Hennessy S, Lewis JD. Insulin therapy and colorectal cancer risk among type 2 diabetes mellitus patients. Gastroenterology 2004;127:1044-50.

6 Chen $\mathrm{K}-\mathrm{H}$, Yeh M-H, Livneh $\mathrm{H}$, et al. Association of traditional Chinese medicine therapy and the risk of dementia in patients with hypertension: a nationwide population-based cohort study. BMC Complement Altern Med 2017:17:178.

7 Tsai T-Y, Livneh H, Hung T-H, et al. Associations between prescribed Chinese herbal medicine and risk of hepatocellular carcinoma in patients with chronic hepatitis $\mathrm{B}$ : a nationwide population-based cohort study. BMJ Open 2017;7:e014571.

8 Tsai T-Y, Li C-Y, Livneh H, et al. Decreased risk of stroke in patients receiving traditional Chinese medicine for vertigo: a populationbased cohort study. J Ethnopharmacol 2016;184:138-43.

9 Kostaki E-G, Nikolopoulos GK, Pavlitina E, et al. Molecular Analysis of Human Immunodeficiency Virus Type 1 (HIV-1)-Infected Individuals in a Network-Based Intervention (Transmission Reduction Intervention Project): Phylogenetics Identify HIV-1-Infected Individuals With Social Links. J Infect Dis 2018;218:707-15.

10 Hao C-zi, Wu F, Lu L, et al. Chinese herbal medicine for diabetic peripheral neuropathy: an updated meta-analysis of 10 high-quality randomized controlled studies. PLoS One 2013;8:e76113.

$11 \mathrm{Xu} \mathrm{H}-\mathrm{B}$, Jiang R-H, Chen X-Z, et al. Chinese herbal medicine in treatment of diabetic peripheral neuropathy: a systematic review and meta-analysis. J Ethnopharmacol 2012;143:701-8.

12 Huang W-J, Fu Q, Xiao Y-H, et al. Effect of qufengtongluo decoction on PI3K/Akt signaling pathway in the kidney of type 2 diabetes mellitus rat (GK rat) with diabetic nephropathy. Evid Based Complement Alternat Med 2018;2018:8421979

13 Liu H, Liu H, Zhou Z, et al. Herbal formula Huang Qin Ge Gen Tang enhances 5-fluorouracil antitumor activity through modulation of the E2F1/TS pathway. Cell Commun Signal 2018;16:7.
14 National health insurance research database, Taiwan. Available: http://nhird.nhri.org.tw/date_cohort.html [Accessed 8 May 2018].

15 Lin T-C, Hwang D-K, Hsu C-C, et al. Protective effect of metformin against retinal vein occlusions in diabetes mellitus - a nationwide population-based study. PLoS One 2017;12:e0188136.

16 Liu CY HY, Chuang YL, Chen YJ, et al. Incorporating development stratification of Taiwan townships into sampling design of large scale health interview survey. $J$ Health Manag 2006;4:1-22.

17 Lai J-N, Hwang J-S, Chen H-J, et al. Finished herbal product as an alternative treatment for menopausal symptoms in climacteric women. J Altern Complement Med 2005;11:1075-84.

18 Lee D-H, Lee TH, Jung CH, et al. Wogonin induces apoptosis by activating the AMPK and p53 signaling pathways in human glioblastoma cells. Cell Signal 2012;24:2216-25.

19 Yao J, Zhao L, Zhao Q, et al. NF-кB and Nrf2 signaling pathways contribute to wogonin-mediated inhibition of inflammationassociated colorectal carcinogenesis. Cell Death Dis 2014;5:e1283

20 Guo P-D, Sun Z-W, Lai H-J, et al. Clinicopathological analysis of PD-L2 expression in colorectal cancer. Onco Targets Ther 2018;11:7635-42.

21 Tu S, Bhagat G, Cui G, et al. Overexpression of interleukin-1 $\beta$ induces gastric inflammation and cancer and mobilizes myeloidderived suppressor cells in mice. Cancer Cell 2008;14:408-19.

22 Xing Z, Xia Z, Peng W, et al. Xuefu Zhuyu decoction, a traditional Chinese medicine, provides neuroprotection in a rat model of traumatic brain injury via an anti-inflammatory pathway. Sci Rep 2016;6:20040.

23 Liu J, Zhang J, Huang L, et al. XuefuZhuyu Tang exerts antitumor effects by inhibiting glioma cell metastasis and invasion via regulating tumor microenvironment. Onco Targets Ther 2016;9:3603-12.

24 Martelli AM, Evangelisti C, Chiarini F, et al. Targeting the PI3K/Akt/ mTOR signaling network in acute myelogenous leukemia. Expert Opin Investig Drugs 2009;18:1333-49.

25 Tong Z, Yu F, Liu Z, et al. Influence of ShuJinHuoXue Tablets on Ischemia Reperfusion Injury of Animals' Skeletal Muscle. Molecules 2012;17:8494-505.

26 Chou CT, Wu WL, Chang ML, et al. Efficacy and possible mechanisms of the Chinese herbs suching-huo-hsuei-tang in the treatment of adjuvant-induced arthritis in rats. Am J Chin Med 1993;21:159-70.

27 Grivennikov SI. Inflammation and colorectal cancer: colitisassociated neoplasia. Semin Immunopathol 2013;35:229-44.

28 Wu C-T, Tsai Y-T, Lin J-G, et al. Chinese herbal products and the reduction of risk of breast cancer among females with type 2 diabetes in Taiwan: a case-control study. Medicine 2018;97:e11600.

29 Sachdev D, Yee D. Disrupting insulin-like growth factor signaling as a potential cancer therapy. Mol Cancer Ther 2007;6:1-12.

30 Yoshida N, Hosokawa T, Ishikawa T, et al. Efficacy of goshajinkigan for oxaliplatin-induced peripheral neuropathy in colorectal cancer patients. J Oncol 2013;2013:139740

31 Korde Choudhari S, Chaudhary M, Bagde S, et al. Nitric oxide and cancer: a review. World J Surg Oncol 2013;11:118.

32 Liu F-C, Pan C-H, Lai M-T, et al. Gan-Lu-Yin inhibits proliferation and migration of murine WEHI-3 leukemia cells and tumor growth in BALB/c allograft tumor model. Evid Based Complement Alternat Med 2013;2013:1-13.

33 Tsai F-J, Ho T-J, Cheng C-F, et al. Characteristics of Chinese herbal medicine usage in ischemic heart disease patients among type 2 diabetes and their protection against hydrogen peroxide-mediated apoptosis in H9c2 cardiomyoblasts. Oncotarget 2017;8:15470-89.

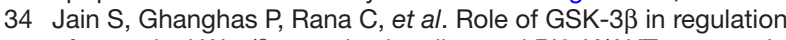
of canonical Wnt/ $\beta$-catenin signaling and PI3-K/AKT oncogenic pathway in colon cancer. Cancer Invest 2017;35:473-83.

35 Chen F-pey, Chen F-jou, Jong M-shiou, et al. Modern use of Chinese herbal formulae from Shang-Han LUN. Chin Med $\mathrm{J}$ 2009;122:1889-94

36 Yang M, Chen J, Xu L, et al. A network pharmacology approach to uncover the molecular mechanisms of herbal formula ban-xia-xiexin-tang. Evid Based Complement Alternat Med 2018;2018:1-22.

37 Zhao L, Zhang S, Wang Z, et al. Efficacy of modified ban Xla xie xin decoction on functional dyspepsia of cold and heat in complexity syndrome: a randomized controlled trial. Evid Based Complement Alternat Med 2013;2013:812143

38 Lakatos P-L, Lakatos L. Risk for colorectal cancer in ulcerative colitis: changes, causes and management strategies. World $\mathrm{J}$ Gastroenterol 2008;14:3937-47. 